\title{
Measurement of the Charge Distribution Along an Electrically Biased Carbon Nanotube using Electron Holography
}

\author{
M. Beleggia,* T. Kasama, * R.E. Dunin-Borkowski,* and G. Pozzi** \\ * Center for Electron Nanoscopy, Technical University of Denmark, DK-2800 Lyngby, Denmark. \\ ** Dipartimento di Fisica, Universita' di Bologna, V.le B. Pichat 6/2, 40127 Bologna, Italy
}

Carbon nanotubes can be examined under an applied bias in the transmission electron microscope in a geometry that is favorable for electron holography and suitable for studying their electrical properties as field emitters. Here we introduce a method that can be used to determine the charge distribution along a biased carbon nanotube from a reconstructed electron holographic phase image. The nanotube is considered to be a one-dimensional object, and in this approximation contributions to the amplitude and phase due to the mean inner potential are neglected. Fig. 1(a,b) shows experimental out-of-focus images of several nanotubes examined in-situ under an applied bias. Fig. 1(c) shows a contour image of the reconstructed phase shift of the nanotube indicated in Fig. 1(b). The out-of-focus contrast, the variation in Fresnel fringe spacing and the change in the apparent diameter of the nanotube suggest that the charge is not uniformly distributed along its length.

The method we propose is based on fundamental laws of electrostatics. Assuming overall charge neutrality, charge $\delta q$ at position $r_{0}$ on a nanotube is associated with equal and opposite induced charge $-\delta q$ on the microscope walls, support grid, specimen holder or substrate. If this induced charge is located at a point $r_{1}$ well outside the field of view, then the phase shift due to the two charges is given by $\varphi=\delta q C_{E} /\left(2 \pi \varepsilon_{0}\right) \log \left(r_{1} / r_{0}\right)$ and is proportional to the two-dimensional electrostatic potential of two lines of charges. Since $-C_{E}^{-1} \nabla \varphi$ is the two-dimensional electric field, according to Gauss' theorem, its flux through a closed surface is exactly equal to the total enclosed charge [1], i.e. $\delta q / \varepsilon_{0}$ at position $r_{0}$. A contour integral of the phase gradient performed around a closed path can, therefore, be used to determine the total charge in the space contained within the loop. The same result is obtained by integrating the Laplacian of the phase within the area bounded by the loop. This method is the electrostatic equivalent of a strategy that we recently proposed for the measurement of magnetic moments from electron holographic phase images [2].

Our proposed approach has far-reaching consequences for the problem at hand, and is independent of whether the image charge is discretely or continuously distributed, so long as the integration loop does not enclose regions in which image charges are present. In addition, if the reference wave is acquired from a vacuum region, then its perturbation is created by charges that are outside the field of view and therefore their field does not influence the measurement of the charge (although it does affect the phase image itself). By the same token, neither any electric field that may influence the reference hologram, nor any linear phase ramps that may be present affect the measured charge. The method is therefore not only able to provide the charge distribution along the nanotube, but it is also, in principle, unaffected by several of the artifacts that usually influence the interpretation of electron holographic phase images.

We have applied our approach to the phase image shown in Fig. 1(c). The results are presented in Fig. 2. Fig. 2(a) shows the phase Laplacian calculated from the rectangular region marked in Fig. 1(c). As the top side of the rectangle is moved upwards, and the values of the pixels within it are 
summed, the antiderivative of the linear charge distribution along the nanotube is obtained. The resulting inferred charge profile shown in Fig. 2(b) reveals two distinct sections of the tube, in which the charge densities are different. A more detailed inspection of the sample confirmed that the diameter of the chosen nanotube changed at a point $\sim 170 \mathrm{~nm}$ from its tip.

The results presented in Fig. 2 are preliminary, and do not take into account possible sources of artifacts such as undersampling, smoothing of the phase image, and noise. Work is in progress to understand these effects, to improve the accuracy of the measurement scheme and to ascertain the conditions under which it is applicable to different specimens [3].

\section{References}

[1] J.D. Jackson, Classical Electrodynamics, 3rd Ed., John Wiley \& Sons (SEA), 1999.

[2] M. Beleggia, T. Kasama, R.E. Dunin-Borkowski, Ultramicroscopy (2010) in press.

[3] We thank Stephan Hofmann (U. Cambridge) for providing the specimen, and for discussions.
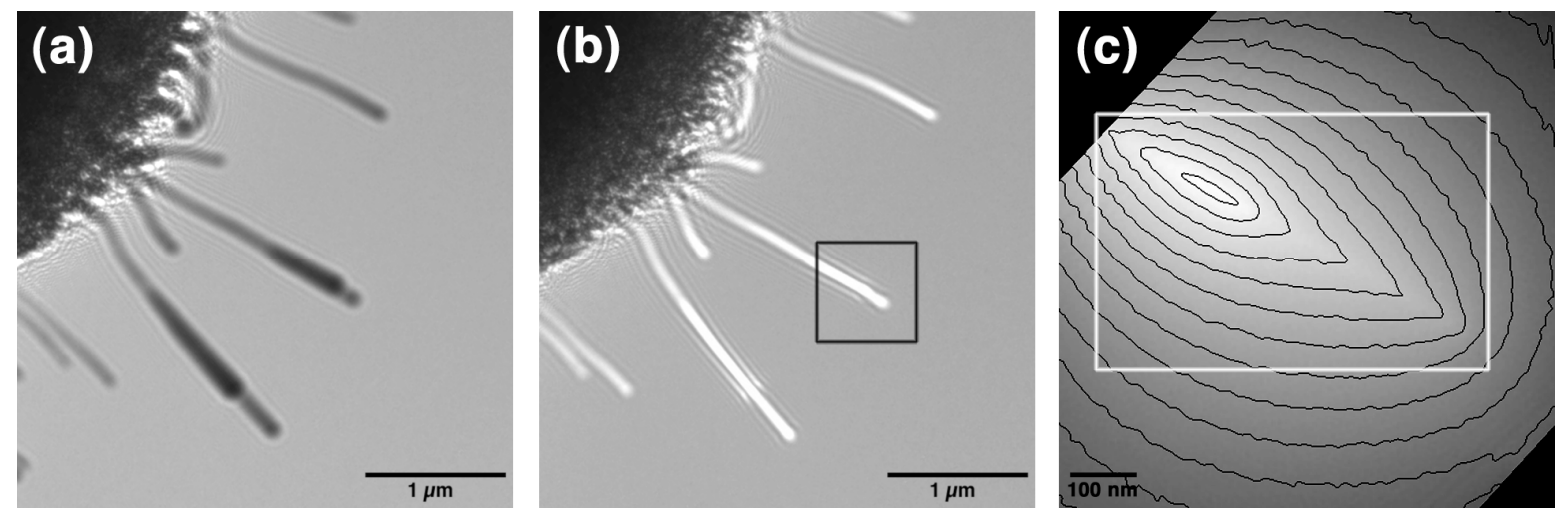

FIG. 1. (a) Underfocus and (b) overfocus images (defocus $\sim 3 \mathrm{~mm}$ ) of carbon nanotubes protruding from a nanotube bundle with an applied bias of $50 \mathrm{~V}$ created by a Au electrode (not shown) $5 \mu \mathrm{m}$ away. (c) Reconstructed phase image acquired from the region indicated in (b), with 1.5 rad phase contours superimposed. The rectangular outline in (c) marks the region shown in Fig. 2(a) below.
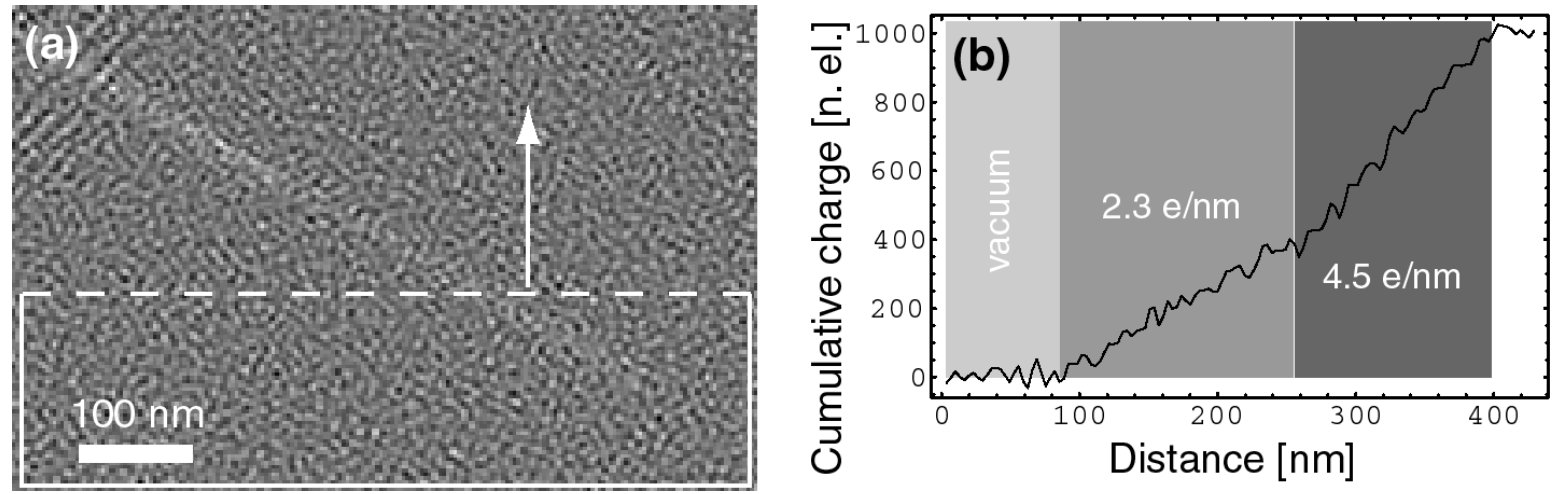

FIG. 2. (a) Laplacian of the phase shift, showing a sketch of the integration procedure. A rectangular region is chosen, with three sides fixed and the fourth (dashed) spanning from bottom to top the entire region: each position of the dashed line yields a single data point, corresponding to the total charge enclosed in the rectangle. (b) Result of the charge measurement revealing three distinct zones: vacuum, with no charge; first section of the nanotube (charge density $\sim 2.3 \mathrm{e} / \mathrm{nm}$ ); second section of the nanotube (charge density $\sim 4.5 \mathrm{e} / \mathrm{nm}$ ). 